\title{
In silico risk assessment for skin sensitization using artificial neural network analysis
}

\author{
Kyoko Tsujita-Inoue, Tomomi Atobe, Morihiko Hirota, Takao Ashikaga and Hirokazu Kouzuki \\ Shiseido Research Center, Shiseido Co. Ltd., 2-2-1 Hayabuchi, Tsuzuki-ku, Yokohama-shi, Kanagawa 224-8558, Japan
}

[Recommended by Takemi Yoshida]

(Received December 11, 2014; Accepted December 26, 2014)

\begin{abstract}
The sensitizing potential of chemicals is usually identified and characterized using in vivo methods such as the murine local lymph node assay (LLNA). Due to regulatory constraints and ethical concerns, alternatives to animal testing are needed to predict the skin sensitization potential of chemicals. For this purpose, an integrated evaluation system employing multiple in vitro and in silico parameters that reflect different aspects of the sensitization process seems promising. We previously reported that LLNA thresholds could be well predicted by using an artificial neural network (ANN) model, designated iSENS ver. 2 (integrating in vitro sensitization tests version 2), to analyze data obtained from in vitro tests focused on different aspects of skin sensitization. Here, we examined whether LLNA thresholds could be predicted by ANN using in silico-calculated descriptors of the three-dimensional structures of chemicals. We obtained a good correlation between predicted LLNA thresholds and reported values. Furthermore, combining the results of the in vitro (iSENS ver. 2) and in silico models reduced the number of chemicals for which the potency category was under-estimated. In conclusion, the ANN model using in silico parameters was shown to be have useful predictive performance. Further, our results indicate that the combination of this model with a predictive model using in vitro data represents a promising approach for integrated risk assessment of skin sensitization potential of chemicals.
\end{abstract}

Key words: Skin sensitization, LLNA, Risk assessment, Artificial neural network, In silico

\section{INTRODUCTION}

Tests for skin sensitization potential are required prior to the introduction of new cosmetic ingredients. Historically, animal models such as the guinea pig maximization test (Magnusson and Kligman, 1969) were used to determine whether a chemical has the potential to induce skin sensitization in humans. More recently, the murine local lymph node assay (LLNA), in which cellular proliferation in the draining lymph node is measured after repeated topical application of the test compound onto the ears, has been employed (Basketter et al., 2002). The relative potency of a chemical sensitizer is measured in terms of the EC3 value, which is the concentration of the test chemical necessary to produce threshold stimulation of proliferation in draining lymph nodes as compared with concurrent vehicle controls (Basketter et al., 2002). Based on EC3 values, chemicals can be classified into five potency categories (extreme, strong, moderate, weak and non-sensitizers), and it is also possible to predict a safe level of human exposure using a quantitative risk assessment approach (Api et al., 2008). However, a recent EU cosmetic directive has prohibited the sale of cosmetic products containing ingredients that have been tested in animals. For this reason, and because of ethical concerns, great efforts have been made to develop alternatives to animal testing to predict the skin sensitization potential of chemicals. Most of these alternative methods use the results of LLNA as in vivo data for prediction. This is since LLNA can provide a relative sensitization potency; information that is pivotal to evaluate the human health risks. Furthermore, it was reported that there was a linear relationship between LLNA EC3 values and human repeated insult patch tests thresholds (Basketter et al., 2005).

Skin sensitization is a complex immunological response consisting of two phases, the induction phase and elicitation phase, and has been well studied over several dec-

Correspondence: Kyoko Tsujita-Inoue (E-mail: kyoko.tsujita@to.shiseido.co.jp) 
ades (Kimber et al., 2002; Aeby et al., 2010; Adler et al., 2011). The mechanisms involved in the induction phase, designated as the adverse outcome pathway (AOP), were reviewed recently in an OECD report (OECD, 2012). Among them, hapten-protein complex formation, production of danger signals and inflammatory mediators by keratinocytes, activation of dendritic cells and T-cell proliferation are recognized as key events. Various in vitro methods focused on these processes have been developed (Gerberick et al., 2007; Suzuki et al., 2009; Natsch and Emter, 2008; Bauch et al., 2012; Emter et al., 2010; Ashikaga et al., 2006; Sakaguchi et al., 2006; Python et al., 2007; Azam et al., 2006; Takahashi et al., 2011; Johansson et al., 2013; Lambrechts et al., 2010; Vacanson et al., 2014). But, because of the complexity of the biological response, it has been recognized that a single in vitro test is insufficient to replace animal testing and that integration of results from different in vitro tests, as well as in silico methods, is needed for prediction of the skin sensitization potential of chemicals (Jowsey et al., 2006; Natsch et al., 2009; Jaworska et al., 2011).

There have also been many reports on prediction of skin sensitization of chemicals by in silico methods (Fedorowicz et al., 2005; Patlewicz et al., 2008; Patlewicz and Worth, 2008). One approach is to use (quantitative) structure-activity relationship ((Q)SAR) models that find mathematical relationships between various physical-chemical or structural properties and biological activity. ADMEWORKS (Fujitsu Kyushu Systems Limited, Fukuoka, Japan), TOPKAT (Accelrys, San Diego, CA, USA), TIMES-SS (University of Bourgas, Bulgaria) and Case Ultra (MULTICASE, Cleveland, OH, USA) are representative QSAR models. Some utilize fragment structures and functional groups considered to be associated with skin sensitization potential as structural alerts in an expert system, as in DEREK for Windows (LHASA Ltd., Leeds, UK). Another approach is readacross, in which chemicals are classified into groups that are considered to exhibit similar or regular patterns of physical-chemical or toxicological properties. Then, the data available for chemicals in the same category may be used for read-across to predict missing toxicological data. The OECD QSAR Toolbox (http://www.oecd.org/ chemicalsafety/risk-assessment/theoecdqsartoolbox.htm) is a representative read-across tool. Recently, Teubner et al. (2013) evaluated 100 chemicals (45 sensitizers and 55 non-sensitizers) using 7 in silico models, including those mentioned above. The best performance was achieved by TIMES-SS, but only $16 \%$ of the test chemicals fell within its reliability domain. There were some models that could provide predictions for almost all compounds, but these gave many false positive and false negative results. They concluded that current in silico models were not sufficiently accurate for broad application to predict skin sensitization potentials.

There have also been attempts to predict the skin sensitization potency of chemicals by using in silico evaluation as part of an integrated testing strategy (Natsch et al., 2009; Jaworska et al., 2011; Nukada et al., 2013). In general, better predictions were obtained when an in silico method was used in combination with other tests in a weight-of-evidence approach, as compared with the use of the other tests alone.

We previously reported that LLNA thresholds (EC3 for LLNA-positive chemicals and maximum test concentrations for LLNA-negative chemicals) could be well predicted by using a combination of in vitro test data focused on protein binding, danger signaling of keratinocytes and activation of dendritic cells in an artificial neural network (ANN) analysis, designated iSENS ver. 2 (integrating in vitro sensitization tests version 2) (Hirota et al., 2013; Tsujita-Inoue et al., 2014). In the present article, we set out to predict the LLNA threshold of chemicals by means of ANN analysis using chemical structure-based determinants obtained by means of molecular orbital (MO) calculations. We also investigated whether the combination of in vitro and in silico ANN models improved the predictive performance for skin sensitization potential.

\section{MATERIALS AND METHODS}

\section{Databases}

We selected a total of 206 chemicals (164 sensitizers and 42 non-sensitizers) (Supplemental data). All the chemicals had previously been evaluated and classified with the LLNA (Gerberick et al., 2005; Basketter et al., 2007; Basketter and Kimber, 2011).

\section{Extraction of descriptors for predicting skin sensitization}

The structures of chemicals were built with Chem 3D Ultra 10.0 (CambridgeSoft Co., Cambridge, MA, USA) and modeled in their neutral forms. Geometry optimization was performed and then molecular-orbital descriptors were calculated using the PM3 Hamiltonian of a semi-empirical MO package (MOPAC2002). The calculated descriptors were as follows: Sum of charges of carbon, hydrogen, oxygen, and nitrogen (Sum C, Sum H, Sum O, Sum N), Core-Core repulsion, COSMO area, COSMO volume, dipole moment, heat of formation, electronic energy, ionization potential, total energy, polarizability $\alpha, \beta, \gamma$, and molecular weight. These descriptors 
were grouped by correlation coefficient, and independent descriptors that were significantly correlated with LLNA thresholds were selected for the ANN input layer.

\section{Artificial neural network (ANN) analysis}

In this study, calculated molecular descriptors and LLNA thresholds were correlated using an artificial neural network. A typical ANN consists of an input layer, a hidden layer, and an output layer, as previously reported (Hirota et al., 2013). The input layer consisted of six molecular descriptors (Sum H, Sum N, heat of formation, ionization potential, polarizability $\beta$, and polarizability $\gamma$ ) that were correlated with LLNA thresholds, and the output layer consisted of LLNA thresholds. We used two hidden layers in this study and all calculations were performed using QwikNet Ver.2.23. A preliminary study indicated that an ANN model with a configuration of 6-42-1 for the input, 1st hidden, 2nd hidden and output layers was optimal in terms of RMS error. An online backpropagation algorithm was used for network training, with values of the learning rate and momentum parameter of 0.02 and 0.7 , respectively. The range of weights was set to be -5 to 5 (weight perturbation; $50 \%$ ).

The predictive ability of the neural network model was evaluated by means of a 10 -fold cross-validation procedure (Hirota et al., 2013). This method systematically removed data successively from the training set. A network model was then constructed on the basis of this reduced data set and was subsequently used to predict the removed data. This procedure was repeated for all data to obtain a complete set of predicted values. The goodness-of-fit was evaluated in terms of root-meansquare (RMS) error, defined as follows:

RMS error $=\sqrt{\left.\sum \text { (measured value }- \text { predicted value }\right)^{2} / \text { number of data }}$

\section{Potency estimation in LLNA and in the ANN prediction model}

Skin sensitization potency classes of chemicals were taken from previous reports (Gerberick et al., 2005; Basketter et al., 2007; Basketter and Kimber, 2011). Sensitization potency was classified into 4 categories: extreme (EC3 in LLNA or predicted LLNA threshold < $0.1 \%$ ), strong (EC3 in LLNA or predicted LLNA threshold, $\geq 0.1 \%$ to $<1 \%$ ), moderate (EC3 in LLNA or predicted LLNA threshold, $\geq 1 \%$ to $<10 \%$ ), and weak or nonsensitizer (EC3 in LLNA or predicted LLNA threshold, $\geq 10 \%$ to $100 \%$ ) in the ANN model.

\section{Statistical analysis}

The correlation coefficient ( $r$ value) was determined by the use of Pearson's correlation statistics. Correlation analysis was performed by Excel Statistics 2004 software (Social Survey Research Information, Tokyo, Japan).

\section{RESULTS}

\section{Extraction of molecular orbital (MO)-calculated descriptors}

LLNA thresholds (EC3 for LLNA-positive chemicals and maximum test concentrations for LLNA-negative chemicals) and MO-calculated descriptors for all compounds were summarized in Supplemental Data. The compounds included 12 extreme, 20 strong, 68 moderate, 65 weak (including 1 false positive) sensitizers and 41 non-sensitizers based on reported LLNA potency classifications (Gerberick et al., 2005; Basketter et al., 2007; Basketter and Kimber, 2011).

The choice of the descriptors is important for ANN analysis. A larger number of descriptors improves the predictive performance in the training data set, but reduces the predictive performance for test compounds because of the vulnerability of the model. Therefore, we classified descriptors having high similarity into groups and selected descriptors that were statistically significantly correlated with LLNA thresholds from independent groups for the ANN input layer. Table 1 shows the simple correlation coefficients between $\mathrm{MO}$-calculated descriptors and LLNA thresholds. Sum H, sum N, heat of formation, ionization potential, polarizability $\beta$ and polarizability $\gamma$ were found to have weak but significant correlations with LLNA thresholds (Table 1, Fig. 1). Then, we classified descriptors with high similarity into groups. Table 2 shows the simple correlation coefficients between MO-calculated descriptors. Correlation coefficients of less than -0.8 or more than 0.8 are highlighted. Sum C, sum $\mathrm{H}, \mathrm{COSMO}$ area and COSMO volume as well as COSMO area, COSMO volume, molecular weight, polarizability $\alpha$, electronic energy and total energy were classified into the same groups because of their high similarity. Finally, we identified 6 apparently independent descriptors (Sum H, sum N, heat of formation, ionization potential, polarizability $\beta$ and polarizability $\gamma$ ) that showed significant correlations with LLNA thresholds, and used these for further ANN analysis.

\section{Establishment of the ANN model for predicting LLNA thresholds}

Using the above descriptors as the input layer and in vivo LLNA thresholds as the output layer in ANN analysis, we obtained a good correlation between predicted and reported values. The correlation coefficient ( $r$ value) 
A

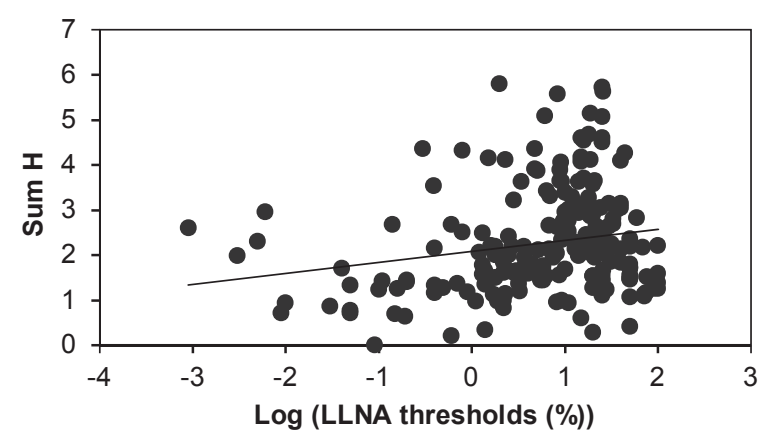

C

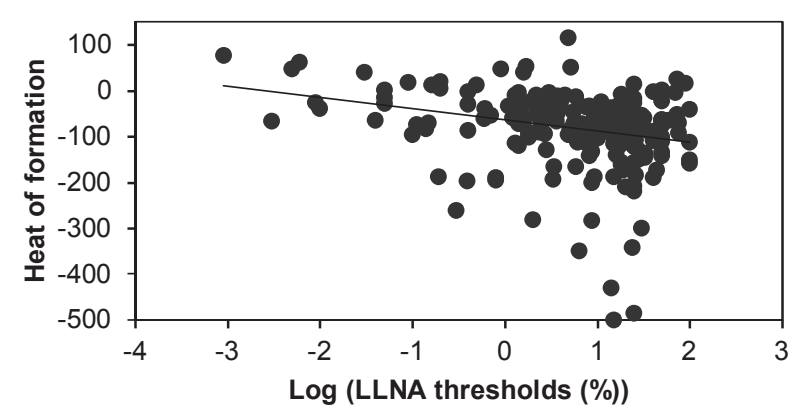

E

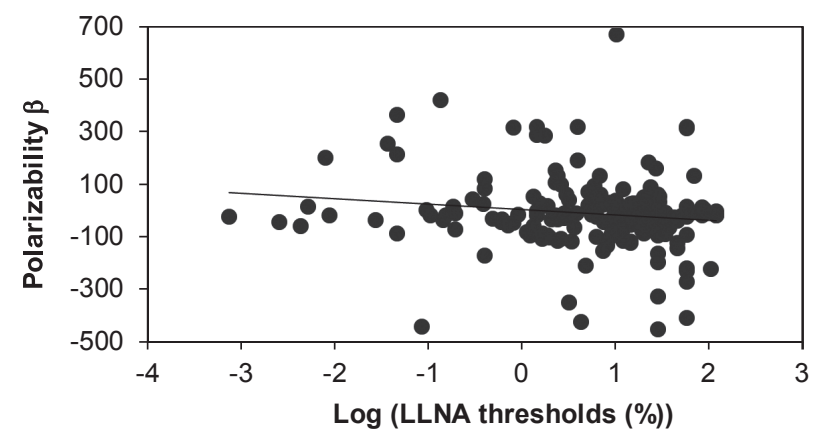

B

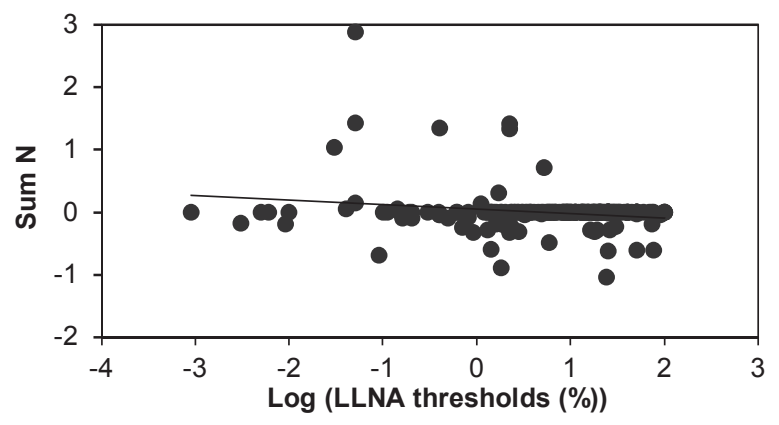

D

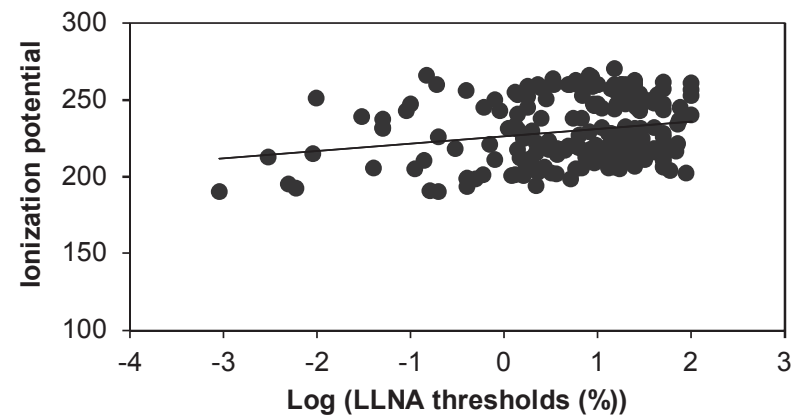

F

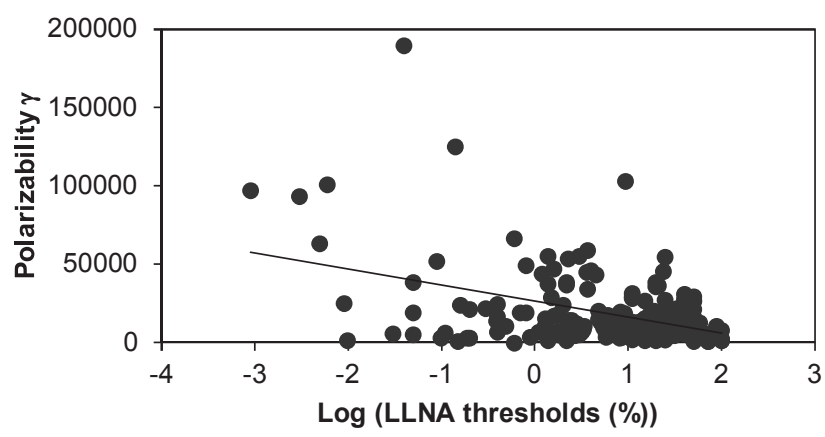

Fig. 1. Relationship between LLNA thresholds and MO-calculated descriptors showing a significant correlation in Table 1. Relationship between LLNA thresholds and Sum H (A), Sum N (B), heat of formation (C), ionization potential (D), polarizability $\beta$ (E) and polarizability $\gamma(\mathrm{F})$.

and root mean square (RMS) error were 0.791 and 0.571 , respectively (Fig. 2A). When we assessed this model by 10 -fold cross-validation $(\mathrm{N}=3)$, the correlation coefficient ( $r$ value) and RMS error were $0.708 \pm 0.018$ and $0.660 \pm 0.017$, respectively (Fig. 2B).

We further classified test samples into 4 potency categories (extreme, strong, moderate and weak sensitizer or non-sensitizer) based on the predicted LLNA thresholds (Table 3A). As shown in Table 3B, accuracy was $55 \%$. The rates of over-prediction and under-prediction of potency categories were $23 \%$ and $22 \%$, respectively.

\section{Integrated evaluation using in vitro and in silico prediction models}

We previously reported that LLNA thresholds could be well predicted with iSENS ver. 2 using a combination of in vitro SH test, ARE assay and h-CLAT, which focus on protein binding, danger signals of keratinocytes and activation of dendritic cells, respectively (TsujitaInoue et al., 2014). In this study, we selected 62 chemi- 
In silico risk assessment for skin sensitization

\begin{tabular}{|c|c|c|c|c|c|c|c|c|c|c|c|c|c|c|c|c|c|c|c|c|}
\hline 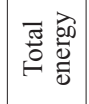 & $\begin{array}{l}\frac{1}{0} \\
\stackrel{0}{0} \\
i\end{array}$ & & & $\begin{array}{l}z \\
\Xi \\
E \\
\omega\end{array}$ & $\begin{array}{l}\infty \\
o ̂ \\
o \\
0 \\
i\end{array}$ & 告 & $\frac{\text { fे }}{0}$ & $\begin{array}{c}\infty \\
\stackrel{0}{0} \\
\stackrel{0}{0} \\
i\end{array}$ & $\begin{array}{l}0 \\
\stackrel{0}{0} \\
\stackrel{0}{0}\end{array}$ & $\begin{array}{l}n \\
\tilde{n} \\
0 \\
i \\
i\end{array}$ & $\begin{array}{c}\stackrel{n}{S} \\
\stackrel{0}{0}\end{array}$ & $\mid \begin{array}{l}0 \\
: \\
0 \\
0 \\
0\end{array}$ & $\left|\begin{array}{l}0 \\
0 \\
d \\
0 \\
i\end{array}\right|$ & 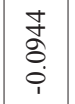 & $\begin{array}{l}\vec{\sigma} \\
\dot{0} \\
i \\
i\end{array}$ & $\mid \begin{array}{l}0 \\
0 \\
0 \\
0\end{array}$ & 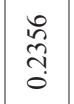 & 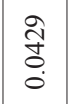 & 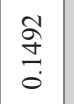 & : \\
\hline 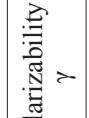 & 晃: & & & 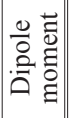 & $\begin{array}{l}0 \\
\text { do } \\
\dot{d} \\
\dot{i}\end{array}$ & $\begin{array}{l}\vec{\infty} \\
\vdots \\
0 \\
0\end{array}$ & $\begin{array}{l}\text { 韋 } \\
\text { 晋 }\end{array}$ & 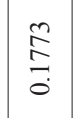 & $\begin{array}{l}\infty \\
\stackrel{2}{0} \\
\stackrel{0}{0}\end{array}$ & $\begin{array}{l}\frac{n}{d} \\
\stackrel{2}{0}\end{array}$ & $\begin{array}{l}\text { o } \\
\text { J } \\
\text { in } \\
\text { i }\end{array}$ & 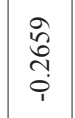 & $\mid \begin{array}{c}\tilde{N} \\
\infty \\
0 \\
0\end{array}$ & $\left|\begin{array}{l}\tilde{n} \\
\tilde{q} \\
\dot{q} \\
i\end{array}\right|$ & $\begin{array}{l}0 \\
: \\
0 \\
0 \\
0 \\
i\end{array}$ & $\left|\begin{array}{c}0 \\
0 \\
0 \\
1 \\
i\end{array}\right|$ & 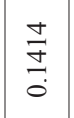 & $\mid \begin{array}{l}\infty \\
0 \\
\\
0 \\
0\end{array}$ & 产 & $\frac{\partial}{\partial}$ \\
\hline$\stackrel{3}{7}$ & $m$ & & & 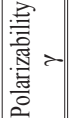 & $\begin{array}{l}0 \\
\stackrel{0}{0} \\
o \\
0 \\
i\end{array}$ & हे & $\stackrel{\infty}{\frac{\infty}{0}}$ & 总 & $\begin{array}{l}\text { Oे } \\
\text { ते } \\
0\end{array}$ & $\mid \begin{array}{l}\hat{a} \\
\bar{n} \\
o\end{array}$ & $\begin{array}{l}\vec{c} \\
\text { ồ } \\
\stackrel{1}{1}\end{array}$ & $\begin{array}{l}2 \\
\stackrel{2}{2} \\
\stackrel{m}{1}\end{array}$ & ल̂. & $\begin{array}{c}\tilde{\infty} \\
\tilde{0} \\
\tilde{O} \\
\dot{i}\end{array}$ & 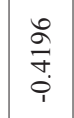 & $\mid \begin{array}{c}0 \\
\stackrel{7}{7} \\
0\end{array}$ & 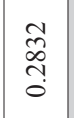 & 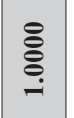 & 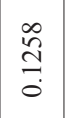 & $\begin{array}{l}\text { సे } \\
\text { ডे. } \\
\text { ¿ }\end{array}$ \\
\hline 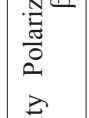 & $=$ & & & 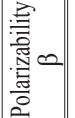 & $\begin{array}{l} \\
\\
0 \\
0 \\
0 \\
1\end{array}$ & 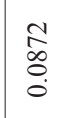 & 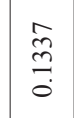 & 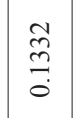 & $\underset{9}{\stackrel{9}{1}}$ & $\frac{\tilde{\sigma}}{0}$ & $\begin{array}{l}\stackrel{\infty}{\circ} \\
\stackrel{m}{-} \\
\stackrel{i}{i}\end{array}$ & $\begin{array}{l}0 \\
0 \\
0 \\
0 \\
\end{array}$ & \begin{tabular}{l|}
0 \\
0 \\
0 \\
0 \\
0
\end{tabular} & 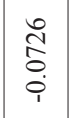 & $\begin{array}{l}\bar{a} \\
\overline{0} \\
\dot{1}\end{array}$ & $\mid \begin{array}{l}n \\
\vdots \\
o \\
0\end{array}$ & 产 & 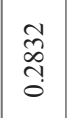 & 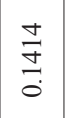 & 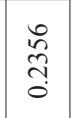 \\
\hline 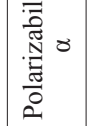 & $\frac{n}{c}$ & & & 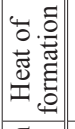 & 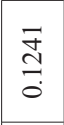 & 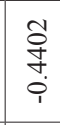 & $\begin{array}{l}8 \\
0 \\
+ \\
0 \\
1\end{array}$ & 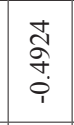 & $\begin{array}{l}0 \\
\dot{0} \\
0 \\
i \\
i\end{array}$ & 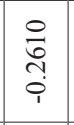 & 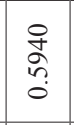 & $\begin{array}{l}\infty \\
0 \\
0 \\
0 \\
0\end{array}$ & 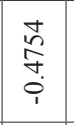 & $\mid$\begin{tabular}{l}
$\infty$ \\
$\mathbb{1}$ \\
\multirow{1}{0}{} \\
0
\end{tabular} & 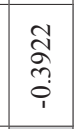 & 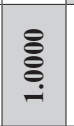 & $\begin{array}{l}n \\
\vdots \\
\vdots \\
0\end{array}$ & $\underset{\widetilde{\pi}}{\stackrel{1}{0}}$ & $\mid \begin{array}{c}0 \\
0 \\
0 \\
0 \\
i \\
i\end{array}$ & $\begin{array}{l}\frac{0}{0} \\
\frac{1}{2} \\
0 \\
0\end{array}$ \\
\hline$z$ & 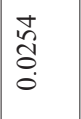 & & & 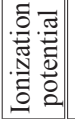 & 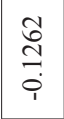 & $\begin{array}{l}\stackrel{0}{2} \\
\stackrel{2}{0}\end{array}$ & 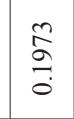 & $\begin{array}{l}\infty \\
\tilde{n} \\
\stackrel{0}{0} \\
0\end{array}$ & $\vec{a}$ & $\begin{array}{l}\mathscr{2} \\
\infty \\
\infty \\
0 \\
i\end{array}$ & $\begin{array}{l}8 \\
\vdots \\
0 \\
i\end{array}$ & $\begin{array}{l}0 \\
0 \\
0 \\
0 \\
0 \\
1\end{array}$ & $\mid \begin{array}{c}\vdots \\
\tilde{J} \\
0 \\
0 \\
i \\
\end{array}$ & $\begin{array}{c}0 \\
0 \\
0 \\
0 \\
1\end{array}$ & 兽 & $\begin{array}{c}\tilde{z} \\
\tilde{\sigma} \\
\tilde{n} \\
i\end{array}$ & $\begin{array}{l}\bar{a} \\
\dot{a} \\
\dot{i}\end{array}$ & $\begin{array}{l}\circ \\
\stackrel{7}{7} \\
\stackrel{0}{0}\end{array}$ & \begin{tabular}{l|}
0 \\
0 \\
0 \\
0 \\
0 \\
$i$
\end{tabular} & $\begin{array}{l}\bar{\sigma} \\
\hat{\sigma} \\
\dot{i}\end{array}$ \\
\hline $\mathbb{N}$ & 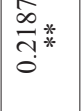 & & & $\begin{array}{l}\circ \\
\Xi \\
\Xi \\
\bar{n}\end{array}$ & 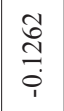 & ֶิ & $\begin{array}{l}\stackrel{2}{\Omega} \\
\stackrel{1}{i}\end{array}$ & 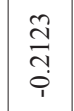 & 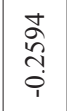 & \begin{tabular}{c}
$\stackrel{\infty}{+}$ \\
$\stackrel{+}{\leq}$ \\
\multirow{1}{*}{}
\end{tabular} & 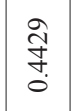 & $\begin{array}{l}n \\
0 \\
f \\
0 \\
0\end{array}$ & $\left|\begin{array}{c}\infty \\
\tilde{N} \\
\tilde{n} \\
i\end{array}\right|$ & 产 & $\begin{array}{l}\stackrel{0}{0} \\
\stackrel{0}{0} \\
\stackrel{i}{1}\end{array}$ & \begin{tabular}{l}
$\infty$ \\
\multirow{2}{*}{} \\
$\stackrel{0}{0}$
\end{tabular} & $\mid \begin{array}{l}0 \\
\mathbb{1} \\
0 \\
0 \\
1\end{array}$ & 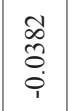 & 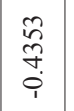 & 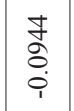 \\
\hline 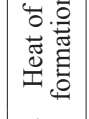 & 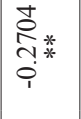 & & & 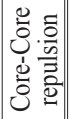 & $\begin{array}{l}\vec{\sigma} \\
\stackrel{\sigma}{\infty} \\
\stackrel{0}{0} \\
i \\
i\end{array}$ & $\begin{array}{l}\text { t. } \\
\stackrel{8}{0} \\
0\end{array}$ & $\begin{array}{l}0 \\
\infty \\
0 \\
0 \\
0 \\
0\end{array}$ & $\mid \begin{array}{l}\tilde{\hat{o}} \\
\stackrel{0}{0} \\
0\end{array}$ & $\begin{array}{l}8 \\
0 \\
0 \\
0\end{array}$ & $\begin{array}{l}n \\
\stackrel{n}{*} \\
0\end{array}$ & $\begin{array}{l}\text { oे } \\
\text { ڤ̆ } \\
\text { iे }\end{array}$ & $\begin{array}{l}\text { ते } \\
\text { o. } \\
0 \\
0\end{array}$ & $\begin{array}{l}\mid \stackrel{8}{\circ} \\
\stackrel{0}{-} \\
\end{array}$ & $\begin{array}{c}\infty \\
\text { ले } \\
\tilde{n} \\
i\end{array}$ & $\begin{array}{l}\mathbb{C} \\
0 \\
0 \\
0 \\
i\end{array}$ & 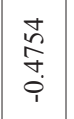 & $\begin{array}{l}n \\
0 \\
0 \\
0 \\
0\end{array}$ & mิ & $\mid \begin{array}{c}n \\
\infty \\
0 \\
0\end{array}$ & 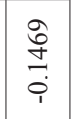 \\
\hline 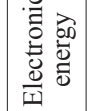 & 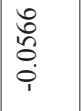 & & & 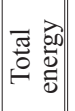 & 总 & $\begin{array}{l}\text { مे } \\
\text { مे } \\
i\end{array}$ & $\begin{array}{l}n \\
\infty \\
\infty \\
0 \\
i\end{array}$ & $\begin{array}{l}\tilde{O} \\
\hat{O} \\
\hat{o}\end{array}$ & $\begin{array}{l}\text { ț } \\
\text { S. } \\
\text { î }\end{array}$ & $\begin{array}{c} \pm \\
0 \\
\infty \\
0 \\
0\end{array}$ & $\begin{array}{l}\tilde{\kappa} \\
\hat{\sigma} \\
\delta\end{array}$ & 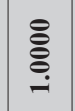 & $\begin{array}{c}\text { Iे } \\
\infty \\
0 \\
i\end{array}$ & $\begin{array}{l}0 \\
0 \\
0 \\
0 \\
0\end{array}$ & $\begin{array}{l}0 \\
0 \\
0 \\
0 \\
\end{array}$ & $\begin{array}{l}\infty \\
0 \\
0 \\
0 \\
0 \\
0\end{array}$ & $\mid \begin{array}{c}0 \\
0 \\
0 \\
0 \\
0\end{array}$ & $\frac{2}{\vec{m}}$ & $\mid \begin{array}{c}0 \\
\hat{0} \\
1 \\
0 \\
i\end{array}$ & $\begin{array}{l}0 \\
\stackrel{n}{5} \\
\vdots \\
0\end{array}$ \\
\hline : & $\infty$ & & $\dot{w}$ & 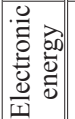 & $\begin{array}{l}0 \\
0 \\
\vdots \\
b \\
0 \\
0\end{array}$ & $\begin{array}{l}\text { त̂ } \\
\text { ลे } \\
\text { i. }\end{array}$ & 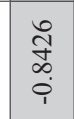 & \begin{tabular}{c}
0 \\
\multicolumn{1}{c}{} \\
$\alpha$ \\
$\dot{1}$
\end{tabular} & 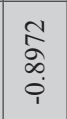 & $\begin{array}{c}8 \\
0 \\
\infty \\
0 \\
1\end{array}$ & 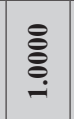 & $\begin{array}{l}n \\
\tilde{s} \\
\vdots \\
0\end{array}$ & $\begin{array}{l}\hat{\sigma} \\
\hat{\sigma} \\
\hat{\sigma} \\
i\end{array}$ & 辛 & $\begin{array}{l} \\
\stackrel{8}{0} \\
\vdots \\
i \\
i\end{array}$ & $\begin{array}{l}\text { 웅 } \\
\text { in } \\
\text { on }\end{array}$ & $\begin{array}{c}\infty \\
0 \\
\stackrel{?}{1} \\
0\end{array}$ & $\begin{array}{l}\overrightarrow{\hat{D}} \\
\stackrel{\infty}{0} \\
\stackrel{1}{1}\end{array}$ & 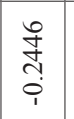 & $\begin{array}{l}\stackrel{m}{0} \\
\stackrel{0}{0}\end{array}$ \\
\hline $\begin{array}{l}25 \\
0 \% \\
0 \%\end{array}$ & 0 & & 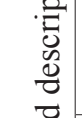 & 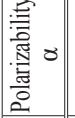 & 菎 & $\begin{array}{l}\text { aे } \\
\stackrel{\infty}{\infty} \\
\stackrel{0}{0}\end{array}$ & 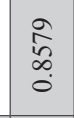 & $\begin{array}{l}n \\
0 \\
\infty \\
\infty \\
0\end{array}$ & $\begin{array}{l}0 \\
\stackrel{2}{0} \\
\alpha \\
0 \\
0\end{array}$ & 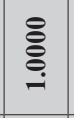 & $\begin{array}{c}8 \\
0 \\
\infty \\
0 \\
1\end{array}$ & \begin{tabular}{|c|} 
\\
0 \\
0 \\
0 \\
0 \\
\end{tabular} & $\begin{array}{l}n \\
\stackrel{n}{5} \\
0\end{array}$ & $\begin{array}{c}\infty \\
\stackrel{0}{ \pm} \\
+ \\
1\end{array}$ & \begin{tabular}{l}
0 \\
$\infty$ \\
$\infty$ \\
\hdashline \\
$i$
\end{tabular} & $\begin{array}{l}0 \\
0 \\
0 \\
i \\
i \\
1\end{array}$ & $\begin{array}{c}\tilde{\sigma} \\
\tilde{\sigma} \\
0\end{array}$ & $\frac{\hat{a}}{\hat{n}}$ & \begin{tabular}{l}
$n$ \\
\multirow{2}{n}{} \\
$\tilde{n}$ \\
0
\end{tabular} & $\begin{array}{l}n \\
\tilde{n} \\
o \\
0 \\
i \\
i\end{array}$ \\
\hline$\widetilde{U}$ & 0 & & 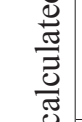 & $\sum$ & $\underset{\vec{i}}{\vec{i}}$ & $\begin{array}{l}\tilde{W} \\
\hat{\delta} \\
0 \\
0\end{array}$ & 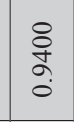 & $\begin{array}{l}\tilde{N} \\
\hat{n} \\
0 \\
0\end{array}$ & 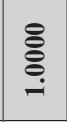 & 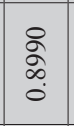 & 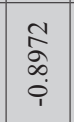 & $\begin{array}{l}\text { to } \\
\text { S̆ } \\
\text { ì }\end{array}$ & 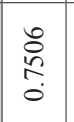 & 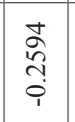 & $\begin{array}{l}\vec{F} \\
0 \\
0 \\
0\end{array}$ & $\begin{array}{l}\vec{j} \\
\dot{j} \\
\dot{i}\end{array}$ & $\mid$\begin{tabular}{c}
9 \\
$\stackrel{9}{ \pm}$ \\
\hdashline \\
0
\end{tabular} & $\begin{array}{l}\text { Oे } \\
\text { ते } \\
0\end{array}$ & $\mid \begin{array}{l}\infty \\
\infty \\
0 \\
0 \\
0\end{array}$ & $\begin{array}{l}\text { o. } \\
\dot{0} \\
\dot{i}\end{array}$ \\
\hline U & & & $\sum_{\overline{0}}^{0}$ & 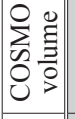 & $\underset{\substack{\hat{\sigma} \\
\infty \\
i \\
i}}{0}$ & \begin{tabular}{l}
\multirow{2}{*}{} \\
ถู
\end{tabular} & $\begin{array}{l}\tilde{\Omega} \\
\text { ઠे }\end{array}$ & 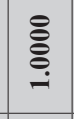 & 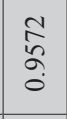 & $\begin{array}{c}n \\
0 \\
\infty \\
\infty \\
0\end{array}$ & 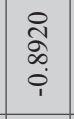 & 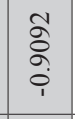 & $\mid \begin{array}{l}n \\
\tilde{0} \\
0 \\
0\end{array}$ & $\begin{array}{c}\tilde{I} \\
\tilde{T} \\
\tilde{i}\end{array}$ & $\begin{array}{l}\infty \\
\stackrel{n}{n} \\
\stackrel{0}{0}\end{array}$ & 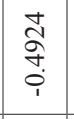 & 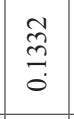 & $\begin{array}{l}\text { ڤัे } \\
\text { ஸे }\end{array}$ & 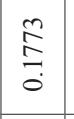 & $\begin{array}{l}\tilde{\infty} \\
\stackrel{0}{O} \\
\vdots \\
\vdots \\
\end{array}$ \\
\hline$\Xi$ & $\tilde{\S}$ & & 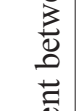 & 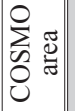 & $\begin{array}{c}n \\
\hat{\infty} \\
\infty \\
i\end{array}$ & $\begin{array}{l}\stackrel{\circ}{\circ} \\
\text { مू } \\
o\end{array}$ & 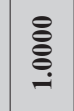 & $\begin{array}{l}\tilde{\Omega} \\
\tilde{\sigma} \\
\hat{\sigma}\end{array}$ & ơ & $\begin{array}{c}\hat{\delta} \\
\hat{\sigma} \\
\infty \\
0\end{array}$ & 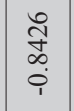 & $\begin{array}{l}n \\
0 \\
\infty \\
\infty \\
0 \\
1\end{array}$ & $\left|\begin{array}{l}\tilde{O} \\
\infty \\
0 \\
0 \\
0\end{array}\right|$ & $\begin{array}{c}m \\
\hat{I} \\
0 \\
1\end{array}$ & $\begin{array}{c}\hat{n} \\
\stackrel{-}{0} \\
\end{array}$ & $\begin{array}{l}8 \\
0 \\
5 \\
0 \\
1\end{array}$ & $\begin{array}{c}\hat{m} \\
\stackrel{m}{0}\end{array}$ & $\frac{\infty}{\frac{\pi}{0}}$ & $\mid \begin{array}{c}\hat{f} \\
\stackrel{5}{0} \\
0\end{array}$ & $\begin{array}{l}\mathcal{I} \\
\stackrel{0}{0} \\
\stackrel{0}{0}\end{array}$ \\
\hline के & 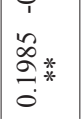 & & $\begin{array}{l}0 \\
\stackrel{0}{0} \\
0 \\
0\end{array}$ & 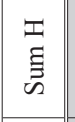 & $\begin{array}{c}\tilde{1} \\
\infty \\
\infty \\
\infty \\
i\end{array}$ & ڤ్ڤ & 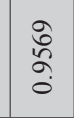 & $\begin{array}{l}\frac{1}{2} \\
\alpha \\
0\end{array}$ & $\begin{array}{l}\widehat{N} \\
0 \\
0 \\
0 \\
0\end{array}$ & $\mid \begin{array}{c}\mathfrak{a} \\
\infty \\
\stackrel{0}{0} \\
0\end{array}$ & 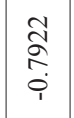 & $\begin{array}{l}2 \\
2 \\
2 \\
i \\
i\end{array}$ & $\begin{array}{l}\text { 苍 } \\
\text { Q } \\
0\end{array}$ & $\begin{array}{l}2 \\
\tilde{a} \\
0 \\
i \\
i\end{array}$ & $\begin{array}{l}\stackrel{\circ}{\swarrow} \\
\stackrel{-}{\circ}\end{array}$ & 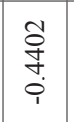 & $\mid$\begin{tabular}{|c|}
$\mathbb{N}$ \\
0 \\
0 \\
0
\end{tabular} & $\begin{array}{l}2 \\
\hat{0} \\
0 \\
0\end{array}$ & $\mid$\begin{tabular}{c|}
$\vec{\infty}$ \\
$\vdots$ \\
0 \\
0
\end{tabular} & 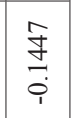 \\
\hline$\exists$ & בิ & $\ddot{\circ}$ & 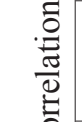 & \begin{tabular}{|l|} 
\\
\\
恶
\end{tabular} & 产 & $\begin{array}{l}\text { N) } \\
\infty \\
\infty \\
i \\
\end{array}$ & $\begin{array}{l}\hat{m} \\
\hat{\infty} \\
\infty \\
i\end{array}$ & $\begin{array}{l}\hat{E} \\
\stackrel{5}{\infty} \\
\dot{i}\end{array}$ & $\underset{i}{\Xi}$ & $\mid \begin{array}{l}0 \\
\overrightarrow{0} \\
0 \\
1\end{array}$ & $\begin{array}{l}0 \\
0 \\
⿱ 亠 䒑 \\
1 \\
0 \\
0\end{array}$ & 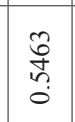 & $\begin{array}{l}\overrightarrow{0} \\
\text { o. } \\
\dot{i}\end{array}$ & 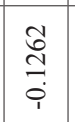 & 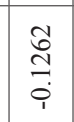 & $\underset{0}{\stackrel{7}{I}}$ & $\begin{array}{l}\text { t. } \\
\text { D. } \\
\text { i. }\end{array}$ & $\begin{array}{l}0 \\
0 \\
0 \\
0 \\
0 \\
i\end{array}$ & 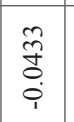 & $\begin{array}{l}\infty \\
0 \\
0 \\
\dot{0} \\
i\end{array}$ \\
\hline 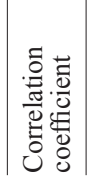 & 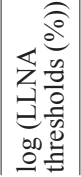 & ? & $\begin{array}{l}\dot{i} \\
\frac{0}{0} \\
\frac{0}{\tilde{\sigma}}\end{array}$ & & $\underline{\Xi}$ & $\stackrel{\bar{B}}{\mathrm{~s}}$ & 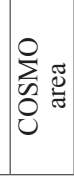 & $\begin{array}{l}x_{2} \\
0 \\
0 \\
0 \\
0\end{array}$ & 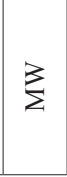 & 8 & 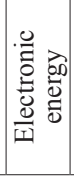 & & 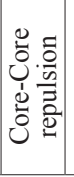 & $\left|\begin{array}{c}0 \\
\vdots \\
\vdots \\
\text { n }\end{array}\right|$ & 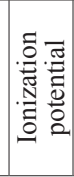 & 苞 & 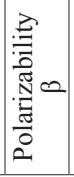 & 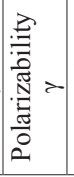 & 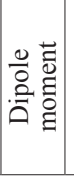 & $\begin{array}{l}z \\
\Xi \\
n \\
\bar{n}\end{array}$ \\
\hline
\end{tabular}


A

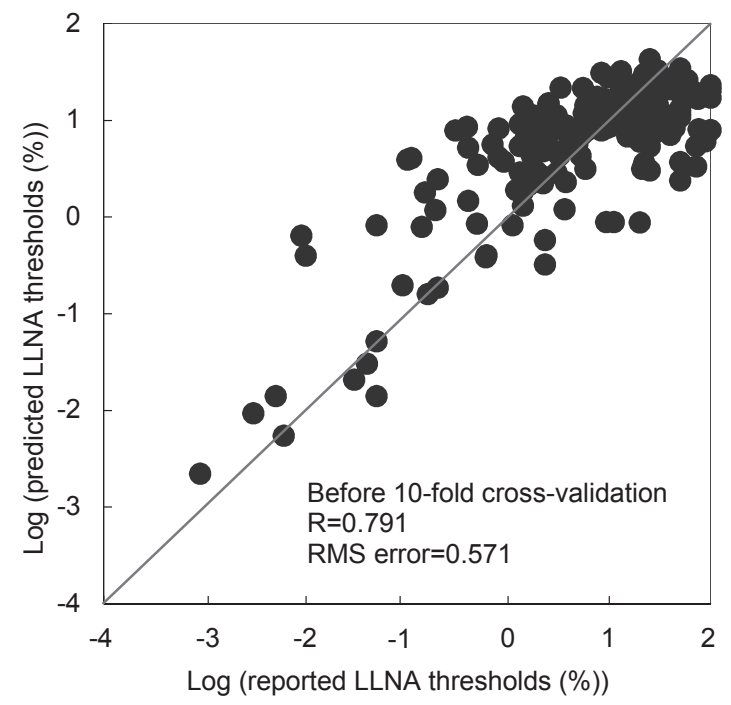

B

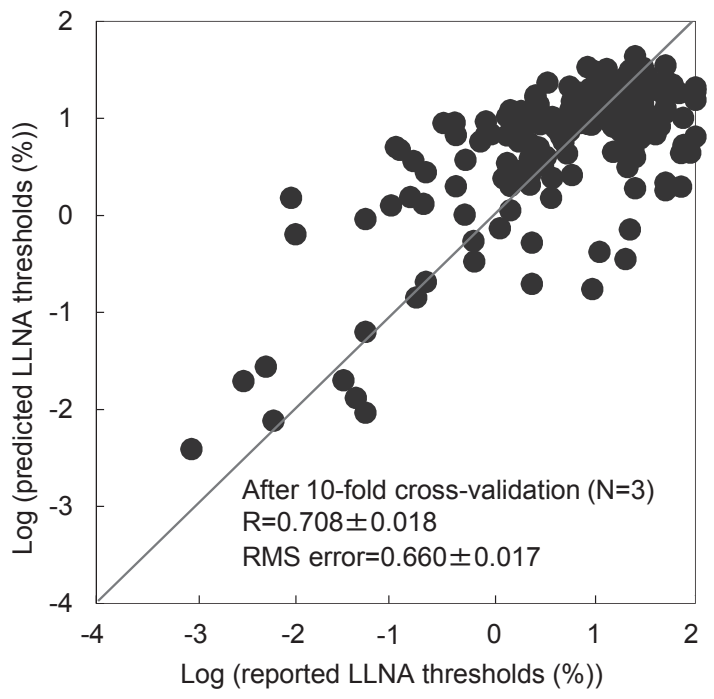

Fig. 2. Correlation between LLNA and ANN prediction results based on MO descriptors of the dataset of 206 chemicals. Six descriptors shown in Fig. 1 were used as the input layer and reported LLNA threshold was used as the output layer. The $r$ values and RMS errors of the ANN prediction model were 0.791 and 0.571 (before cross-validation (A)) and $0.708 \pm 0.018$ and $0.660 \pm 0.017$ (after cross-validation $(\mathrm{B})(\mathrm{N}=3)$ ).

cals from the iSENS ver. 2 dataset (Table 4A). It was not possible to obtain MO-calculated descriptors of metalcontaining chemicals (potassium dichromate, cobalt chloride and nickel sulfate) with MOPAC 2002. Fig. 3 compares the predicted and reported LLNA thresholds. For the 53 chemicals in the training dataset we used the predicted LLNA thresholds obtained after 10-fold cross-validation, and for the other 6 (diphenylcyclopropenone, phthalic anhydride, maleic anhydride, benzoyl peroxide, propyl gallate, and 4-chloroaniline) we used the predicted thresholds. The in silico model gave a good correlation between predicted and reported LLNA thresholds for these 59 chemicals (Fig. 3B). As shown in Fig. 3A and $\mathrm{B}$, about half the chemicals showed predicted LLNA thresholds higher than the reported values, which indicates that skin sensitization potency is underestimated. From the viewpoint of safety assurance, it is important to reduce the number of under-estimated chemicals. Therefore, we adopted the lower of the predicted LLNA thresholds in the two models and compared the resulting values with reported data (Fig. 3C). In the combined model, all the predicted LLNA thresholds were the same as or less than in iSENS ver. 2. The predictivity of iSENS ver. 2 , the in silico model and the combined model is summarized in Table 4B. It should be noted that under-prediction of potency category was reduced to $8 \%$ with the combination of the in vitro (iSENS ver. 2) and in silico models.

\section{DISCUSSION}

We previously reported that LLNA threshold as a continuous value was well predicted using descriptors derived from in vitro test results by ANN analysis with our systems iSENS ver. 1 and iSENS ver. 2 (Hirota et al., 2013; Tsujita-Inoue et al., 2014). In the AOP of skin sensitization (OECD, 2012), initial molecular events such as protein-binding and cellular responses of keratinocytes and dendritic cells play important roles. Thus, the descriptors in these ANN models were selected from tests considered to represent the AOP key events. On the other hand, it is well known from practical experience that certain kinds of chemical structure are associated with skin sensitization potential. For example, Schiff base or Michael addition functionality tends to be associated with skin sensitization. Indeed, the knowledge-based DEREK system employs structural alerts. However, it would be difficult to quantitatively predict skin sensitization potency on the basis of structural alerts. Therefore, we aimed to construct an ANN model for predicting LLNA thresholds by using structure-derived molecular orbital (MO)-calcu- 
In silico risk assessment for skin sensitization

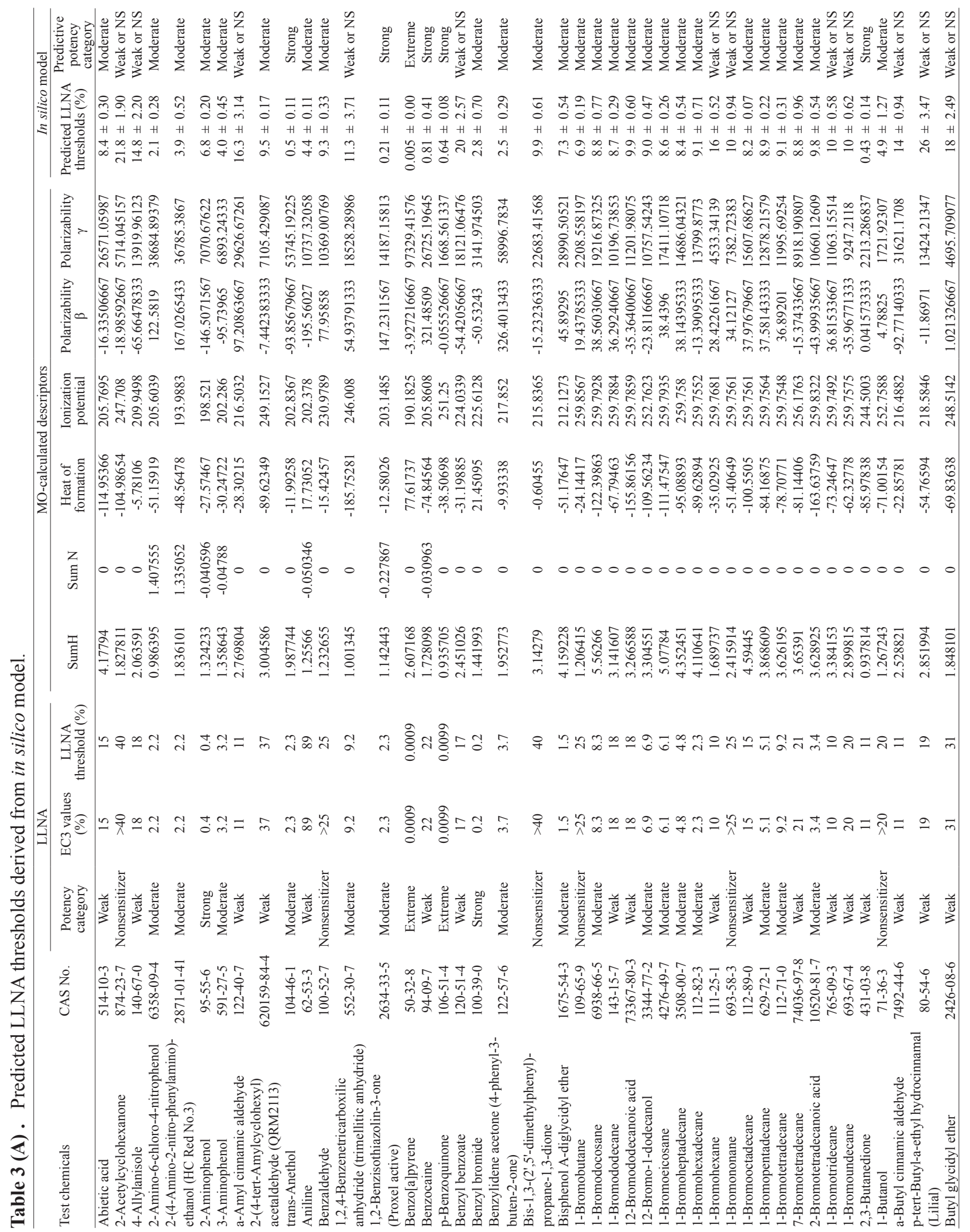


K. Tsujita-Inoue et al.

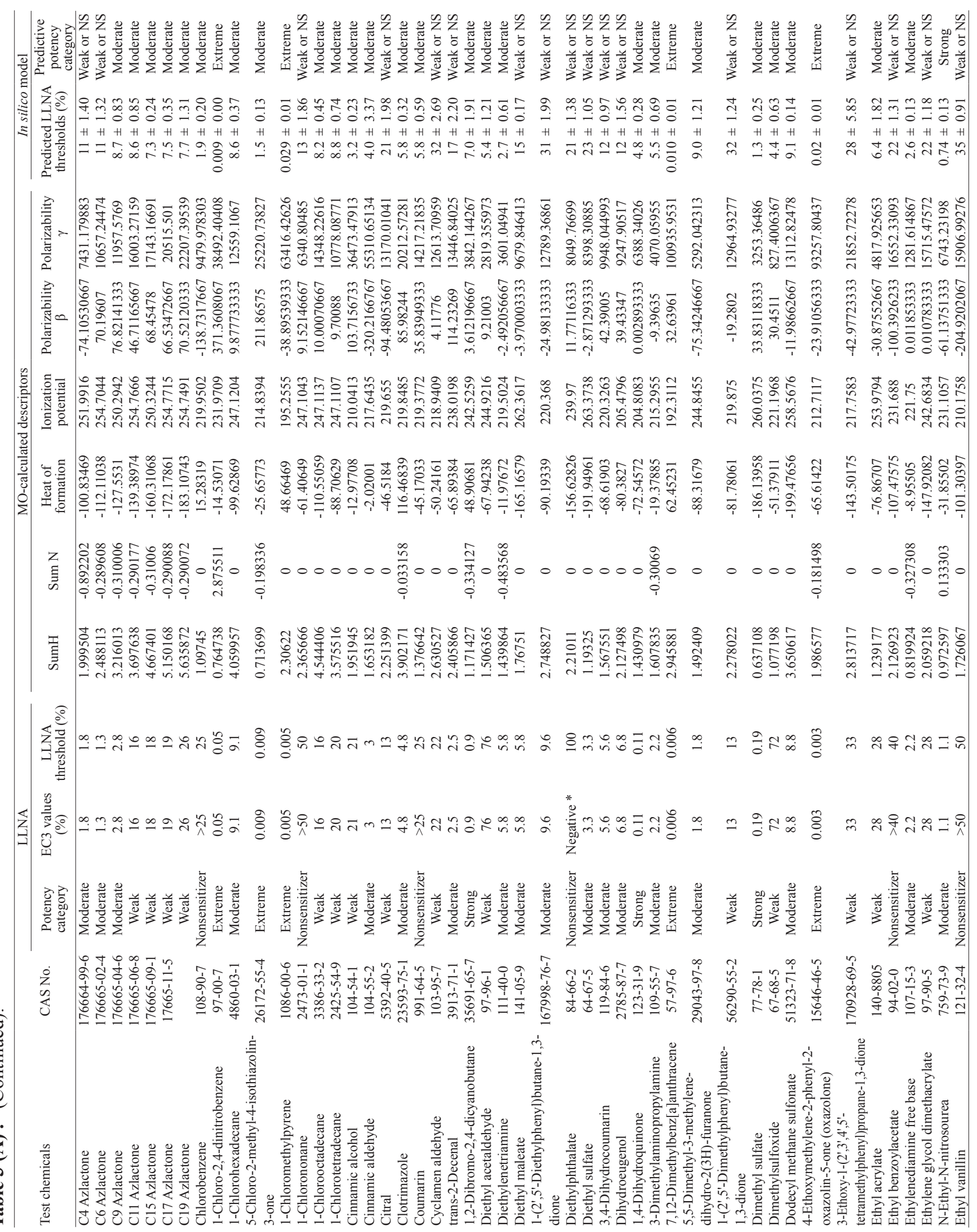

Vol. 40 No. 2 
In silico risk assessment for skin sensitization

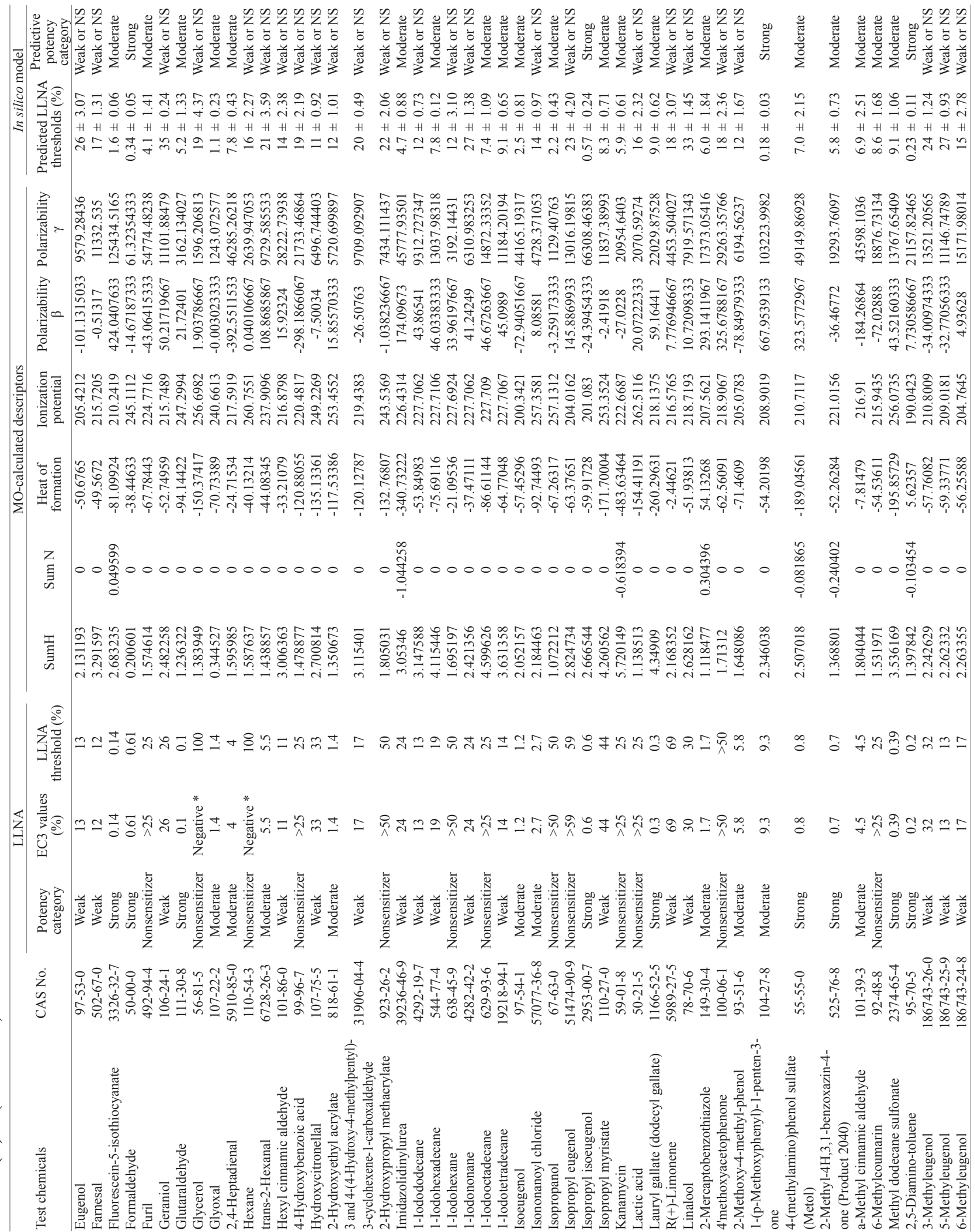




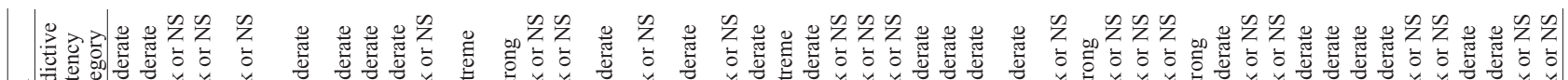

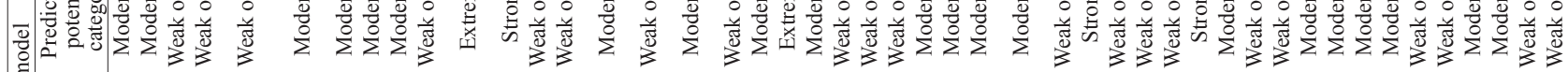

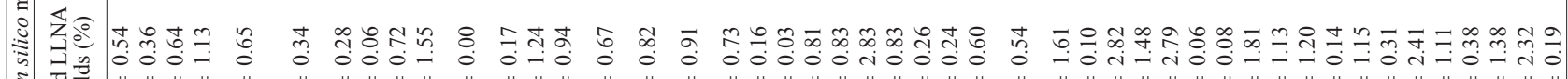

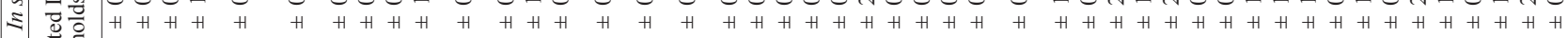

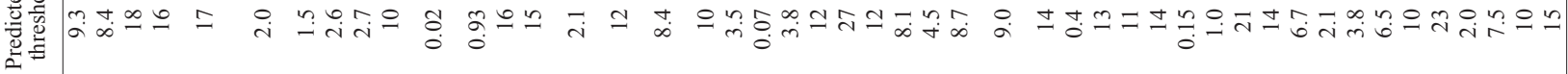

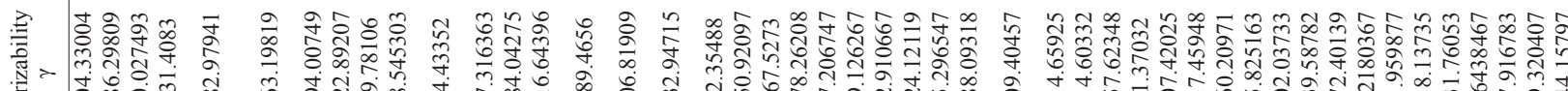
焉

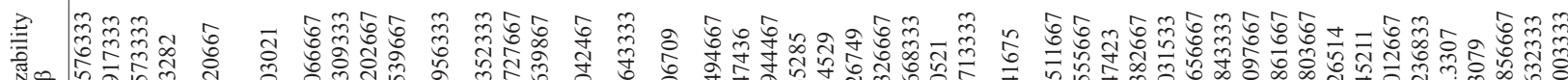
第

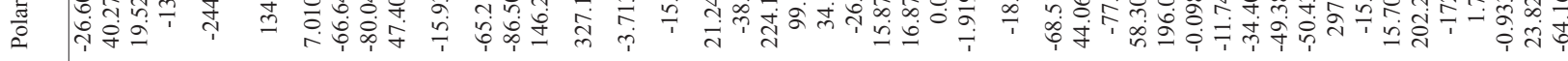

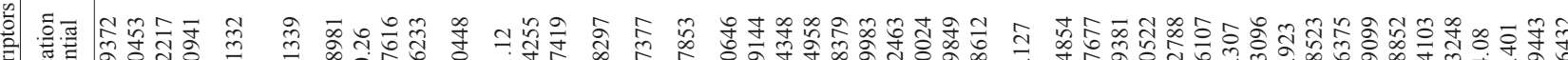

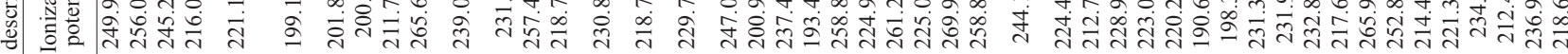

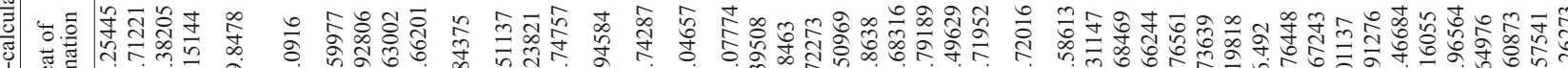

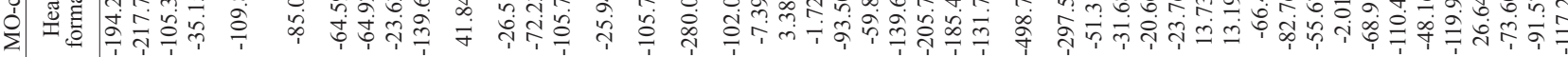

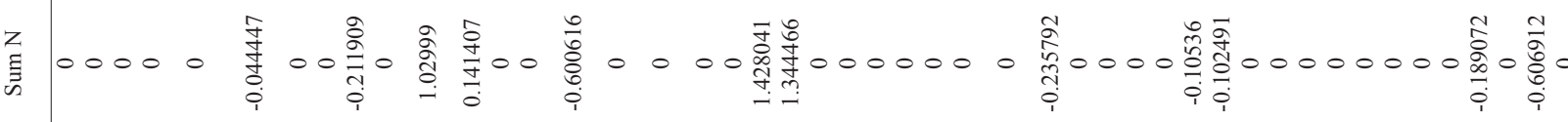

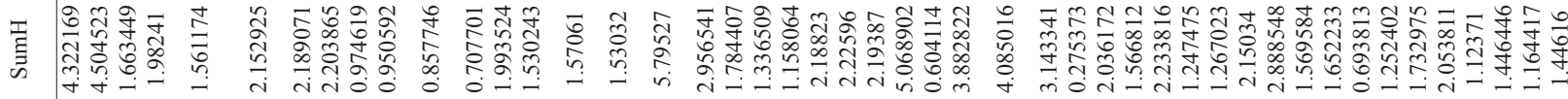

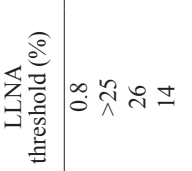

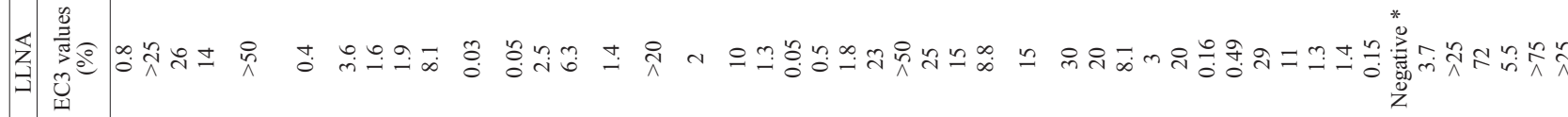

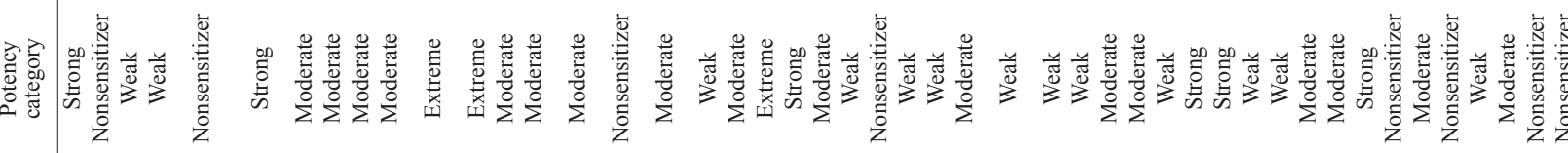

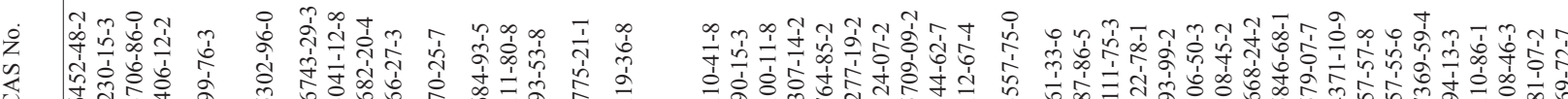

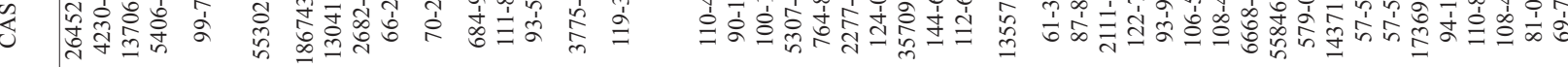

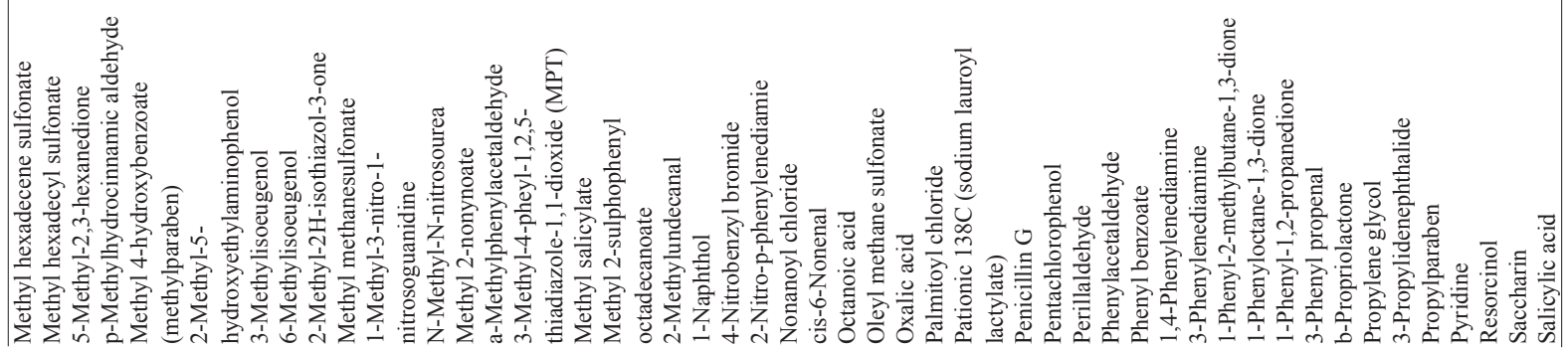



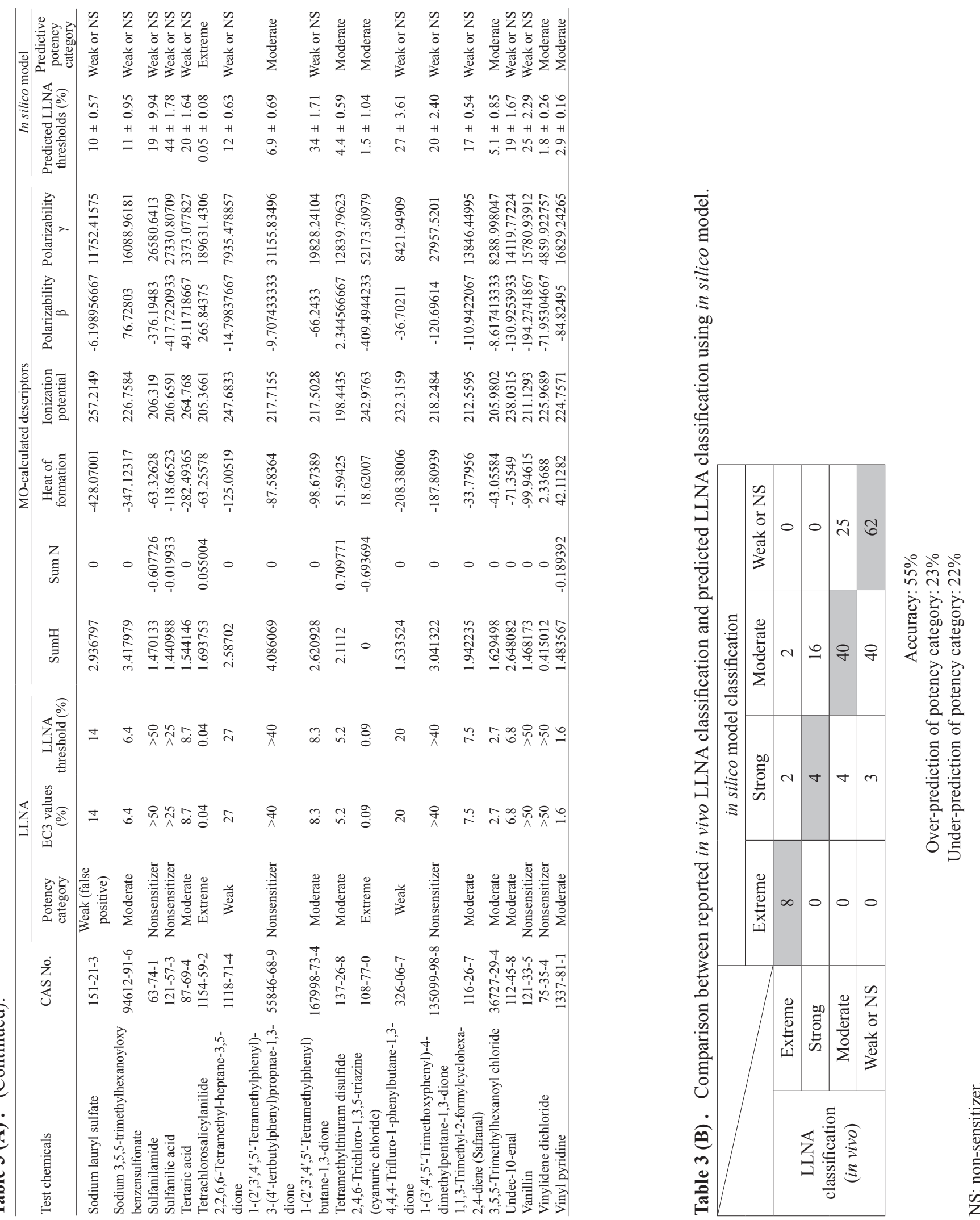
K. Tsujita-Inoue et al.

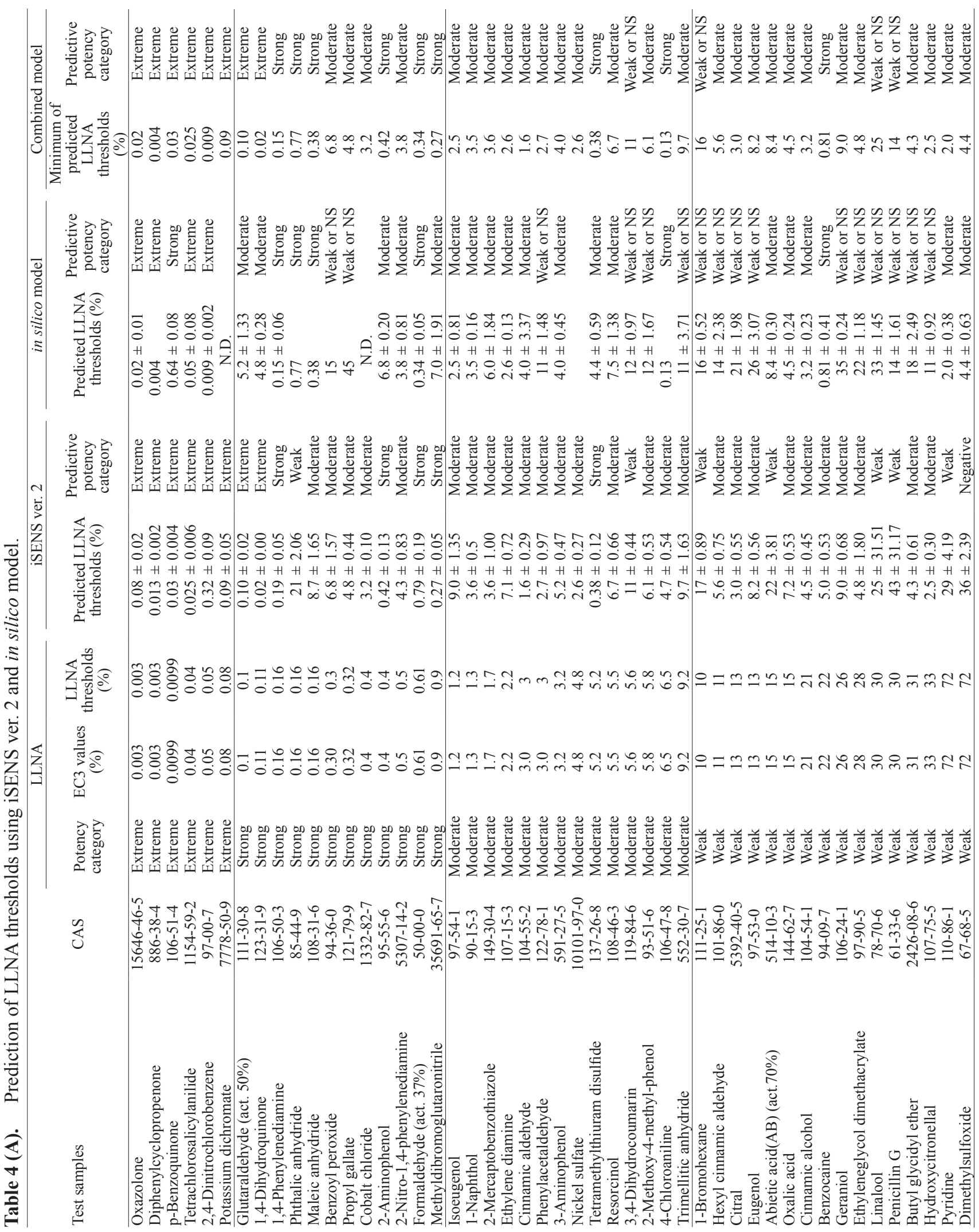

Vol. 40 No. 2 
In silico risk assessment for skin sensitization
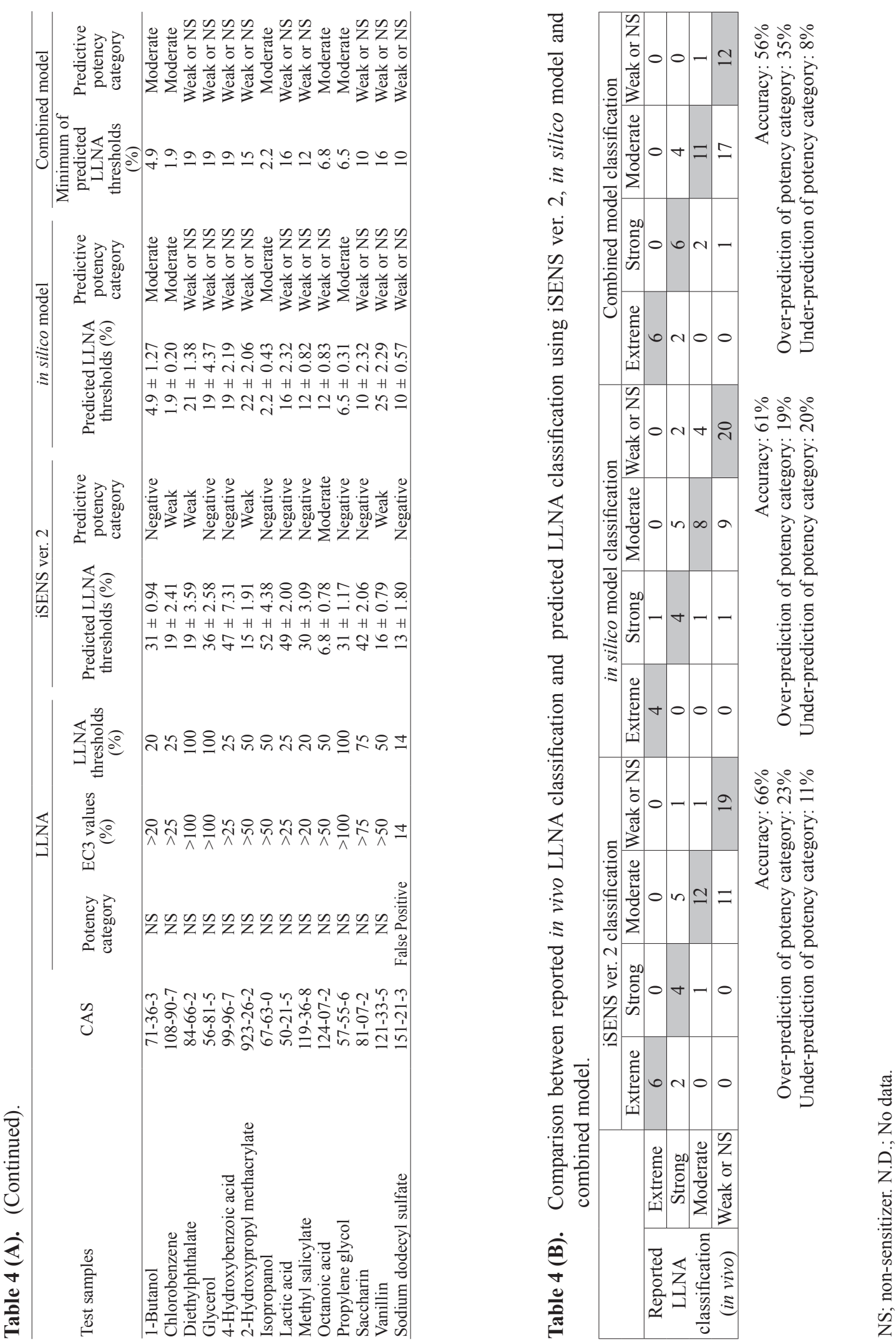
A

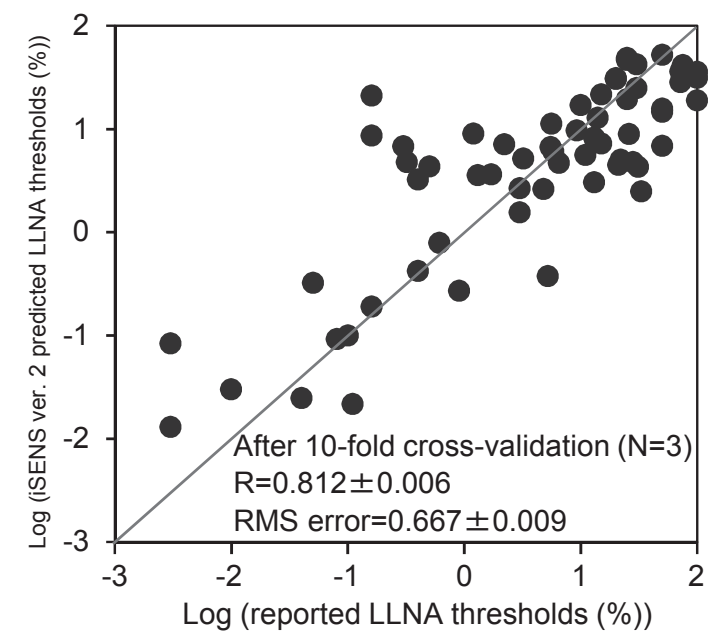

C

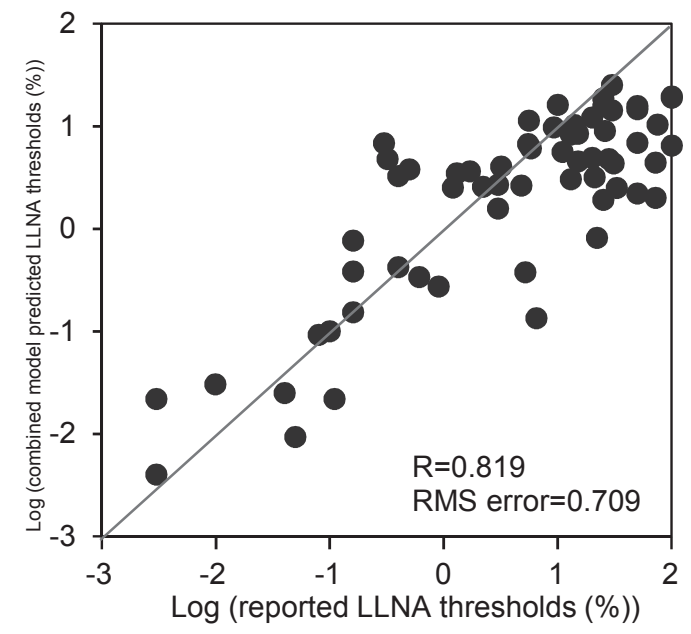

B

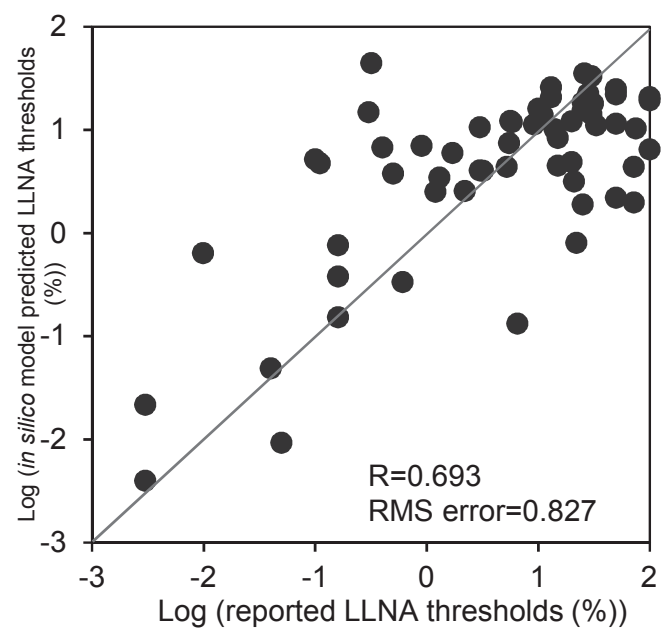

Fig. 3. Correlation between LLNA and ANN prediction resuls of iSENS ver. 2 and in silico model. (A) ANN prediction results of iSENS ver. 2 for 62 chemicals reported previously. (B) ANN prediction results of in silico model for 59 chemicals. (C) Correlation between reported LLNA threshold and minimum of (predicted LLNA threshold of iSENS ver. 2 and in silico model).

lated descriptors in place of structural alerts. We selected Sum H, Sum N, heat of formation, ionization potential, polarizability $\beta$ and polarizability $\gamma$ as independent, statistically significant descriptors and showed that ANN using these descriptors enables us to predict skin sensitization potency of chemicals. The reason why this approach works may be that the descriptors are related to physicochemical properties known to be important for skin sensitization.

For example, liposolubility is related to polarizability, molecular volume and hydrogen bonding property, and so it is reasonable to consider that the relevant descriptors (polarizability $\beta$, polarizability $\gamma$, Sum $H$, Sum N and ionization potential) are correlated with skin absorption and binding affinity to protein. Sum H, Sum N and ionization potential are also related to electronegativity, and therefore to nucleophilic and electrophilic reactivity. On the other hand, ionization potential, heat of formation, and total energy are expected to influence the stability of chemicals, and so might be related to metabolic degradability. Therefore, the descriptors selected in this study are likely to reflect biological processes that are important in 
skin sensitization.

The ITS concept integrates various types of in vitro and in silico information, including biological, biochemical and chemical factors that impact on the allergenic properties of chemicals and the acquisition of skin sensitization potency, for prediction of skin sensitization potential (Jowsey et al., 2006). Several studies have reported hazard identification or potency categorization of skin sensitization by means of majority vote or scorebased systems or a Bayesian network (Bauch et al., 2012; Natsch et al., 2013; Nukada et al., 2013; Jaworska et al., 2011, 2013). In the present study, we performed ANN analysis with in silico descriptors, and integrated the predictions of iSENS ver. 2 and this in silico model. Since it is important to reduce the number of under-estimations from the viewpoint of safety assurance, we adopted the lower of the values predicted by these two models as the LLNA threshold of the combined model. As shown in Table 4B, 7 chemicals were under-predicted in potency category classification by iSENS ver. 2. Among them, it was not possible to calculate MO descriptors of cobalt chloride, as previously mentioned. For benzoyl peroxide, propyl gallate and 3,4-dihydrocoumarin, the predicted LLNA thresholds were higher in the in silico model than in iSENS ver. 2, so that they were still under-predicted by the combined model. For phthalic anhydride, maleic anhydride and 2-nitro-1,4-phenylenediamine, the predicted LLNA thresholds were lower in the in silico model than in iSENS ver. 2. As a result, the predicted potency categories of these chemicals became consistent with the reported category in LLNA, except in the case of 2-nitro1,4-phenylenediamine, which was still under-predicted in the combined model. Phthalic anhydride, which was reported to be strong sensitizer in LLNA, was positive in the SH test, but negative in h-CLAT and ARE assay. In iSENS ver. 2, it was predicted as a weak sensitizer. Thus, the combination of in vitro and in silico models is expected to be very useful for chemicals that are false negative in some in vitro studies. As described above, the number of under-estimated chemicals was decreased with the combined model (Table 4B). When evaluating chemicals without animal testing, comprehensive risk assessment before application to humans is extremely important, especially for sensitizing materials. Therefore, there is a strong requirement for the combined model to minimize the number of under-estimated chemicals, and the issue of over-estimated chemicals may be less critical. Among 10 chemicals predicted as non-sensitizers in iSENS ver. 2 , only dimethylsulfoxide and sodium dodecyl sulfate were false negatives. Dimethylsulfoxide was rated a weak sensitizer in LLNA with a high EC3 of 77\%, while sodi-

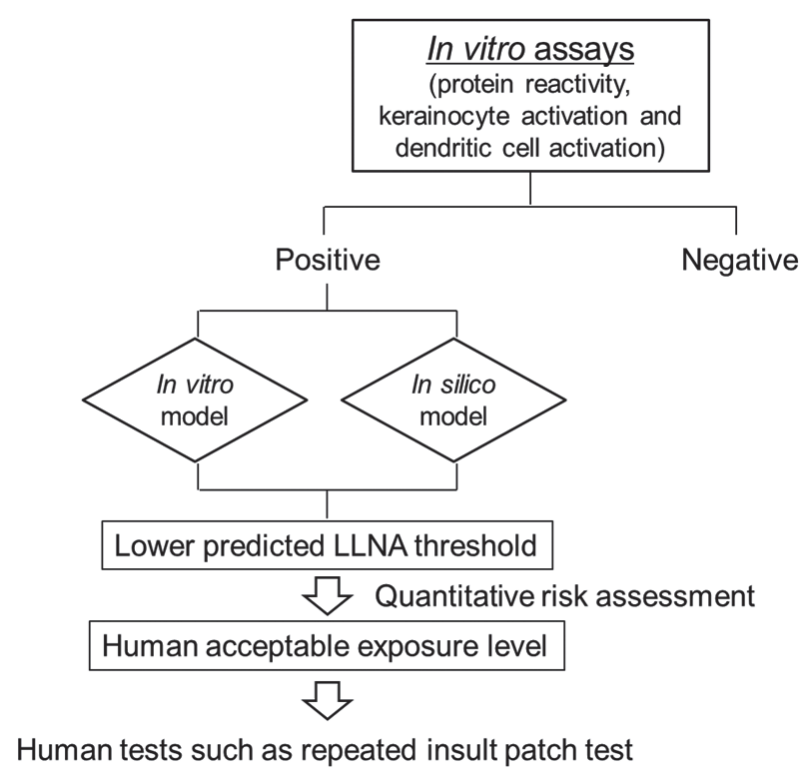

Fig. 4. Proposed evaluation scheme for skin sensitization risk using our ANN model.

um dodecyl sulfate was rated as false positive in LLNA. Since iSENS ver. 2 generates very few false negatives, it may be a realistic approach to apply the combined model including the ANN system only to chemicals predicted as positive by iSENS ver. 2, as shown in Fig. 4.

In conclusion, our ANN model using descriptors calculated from chemical structures was effective to predict LLNA thresholds. This is a new approach for in silico prediction, because most previous in silico models aimed at predicting skin sensitization were constructed only for hazard identification. The combination of our previous ANN model using in vitro data with the present in silico model appears to be an effective approach for predicting sensitizing potency. Furthermore, our ANN models can be used to calculate an acceptable human exposure level, using a quantitative risk assessment approach (Api et al., 2008). Our results indicate that further development of combined in vitro and in silico tools for non-animal risk assessment of skin sensitization would be worthwhile.

\section{ACKNOWLEDGMENT}

We thank Richard Steele for a critical review of the manuscript.

Conflict of interest---- The authors declare that there is no conflict of interest. 


\section{REFERENCES}

Adler, S., Basketter, D., Creton, S., Pelkonen, O., van Benthem, J., Zuang, V., Andersen, K.E., Angers-Loustau, A., Aptula, A., Bal-Price, A., Benfenati, E., Bernauer, U., Bessems, J., Bois, F.Y., Boobis, A., Brandon, E., Bremer, S., Broschard, T., Casati, S., Coecke, S., Corvi, R., Cronin, M., Daston, G., Dekant, W., Felter, S., Grignard, E., Gundert-Remy, U., Heinonen, T., Kimber, I., Kleinjans, J., Komulainen, H., Kreiling, R., Kreysa, J., Leite, S.B., Loizou, G., Maxwell, G., Mazzatorta, P., Munn, S., Pfuhler, S., Phrakonkham, P., Piersma, A., Poth, A., Prieto, P., Repetto, G., Rogiers, V., Schoeters, G., Schwarz, M., Serafimova, R., Tähti, H., Testai, E., van Delft, J., van Loveren, H., Vinken, M., Worth, A. and Zaldivar, J.M. (2011): Alternative (non-animal) methods for cosmetics testing: current status and future prospects-2010. Arch. Toxicol., 85, 367-485.

Aeby, P., Ashikaga, T., Bessou-Touya, S., Schepky, A., Gerberick, F., Kern, P., Marrec-Fairley, M., Maxwell, G., Ovigne, J.M., Sakaguchi, H., Reisinger, K., Tailhardat, M., MartinozziTeissier, S. and Winkler, P. (2010): Identifying and characterizing chemical skin sensitizers without animal testing: Colipa's research and method development program. Toxicol. In Vitro, 24, 1465-1473.

Api, A.M., Basketter, D.A., Cadby, P.A., Cano, M.F., Ellis, G., Gerberick, G.F., Griem, P., McNamee, P.M., Ryan, C.A. and Safford, R. (2008): Dermal sensitization quantitative risk assessment (QRA) for fragrance ingredients. Regul. Toxicol. Pharm., 52, 3-23.

Ashikaga, T., Yoshida, Y., Hirota, M., Yoneyama, K., Itagaki, H., Sakaguchi, H., Miyazawa, M., Ito, Y., Suzuki, H. and Toyoda, H. (2006): Development of an in vitro skin sensitization test using human cell lines: the human cell line activation test (h-CLAT). I. Optimization of the h-CLAT protocol. Toxicol. In Vitro, 20, 767-773.

Azam, P., Peiffer, J.L., Chamousset, D., Tissier, M.H., Bonnet, P.A., Vian, L., Fabre, I. and Ourlin, J.C. (2006): The cytokine-dependent MUTZ-3 cell line as an in vitro model for the screening of contact sensitizers. Toxicol. Appl. Pharmacol., 212, 14-23.

Basketter, D.A., Clapp, C., Jefferies, D., Safford, B., Ryan, C.A., Gerberick, F., Dearman, R.J. and Kimber, I. (2005): Predictive identification of human skin sensitization thresholds. Contact Dermatitis, 53, 260-267.

Basketter, D.A., Evans, P., Fielder, R.J., Gerberick, G.F., Dearman, R.J. and Kimber, I. (2002): Local lymph node assay - validation, conduct and use in practice. Food Chem. Toxicol., 40, 593-598.

Basketter, D.A. and Kimber, I. (2011): Predictive tests for irritants and allergens and their use in quantitative risk assessment. In "Contact Dermatitis", 5th edition, chapter 13. Springer, Berlin, Eds Johansen, J.D., Frosch, P.F. and Lepoittevin, J-P., pp.229240.

Basketter, D.A., Sanders, D. and Jowsey, I.R. (2007): The skin sensitization potential of resorcinol: Experience with the local lymph node assay. Contact Dermatitis, 56, 196-200.

Bauch, C., Kolle, S.N., Ramirez, T., Eltze, T., Fabian, E., Mehling, A., Teubner, W., van Ravenzwaay, B. and Landsiedel, R. (2012): Putting the parts together: combining in vitro methods to test for skin sensitizing potentials. Regul. Toxicol. Pharm., 63, 489-504.

Emter, R., Ellis, G. and Natsch, A. (2010): Performance of a novel keratinocyte-based reporter cell line to screen skin sensitizers in vitro. Toxicol. Appl. Pharmacol., 245, 281-290.

Fedorowicz, A., Singh, H., Soderholm, S. and Demchuk, E. (2005):
Structure-activity models for contact sensitization. Chem. Res. Toxicol., 18, 954-969.

Gerberick, G.F., Ryan, C.A., Kern, P.S., Schlatter, H., Dearman, R.J., Kimber, I. Patlewicz, G.Y. and Basketter, D.A. (2005): Compilation of historical local lymph node data for evaluation of skin sensitization alternative methods. Dermatitis, 16, 157202.

Gerberick, G.F., Vassallo, J.D., Foertsch, L.M., Price, B.B., Chaney, J.G. and Lepoittevin, J.P. (2007): Quantification of chemical peptide reactivity for screening contact allergens: a classification tree model approach. Toxicol. Sci., 97, 417-427.

Hirota, M., Kouzuki, H., Ashikaga, T., Sono, S., Tsujita, K., Sasa, H. and Aiba, S. (2013): Artificial neural network analysis of data from multiple in vitro assays for prediction of skin sensitization potency of chemicals. Toxicol. In Vitro, 27, 1233-1246.

Jaworska, J., Harol, A., Kern, P.S. and Gerberick, G.F. (2011): Integrating non-animal test information into an adaptive testing strategy - skin sensitization proof of concept case. ALTEX, 28, 211-225.

Jaworska, J., Dancik, Y., Kern, P., Gerberick, F. and Natsch, A. (2013): Bayesian integrated testing strategy to assess skin sensitization potency: from theory to practice. J. Appl. Toxicol., 33, 1353-1364.

Johansson, H., Albrekt, A.S., Borrebaeck, C.A. and Lindstedt, M. (2013): The GARD assay for assessment of chemical skin sensitizers. Toxicol. In Vitro, 27, 1163-1169.

Jowsey, I.R., Basketter, D.A., Westmoreland, C. and Kimber, I. (2006): A future approach to measuring relative skin sensitising potency: a proposal. J. Appl. Toxicol., 26, 341-350.

Kimber, I., Basketter, D.A., Gerberick, F. and Dearman, R.J. (2002): Allergic contact dermatitis. Int. Immunopharmacol., 2, 201-211.

Lambrechts, N., Vanheel, H., Nelissen, I., Witters, H., Van Den Heuvel, R., Van Tendeloo, V., Schoeters, G. and Hooyberghs, J. (2010): Assessment of chemical skin-sensitizing potency by an in vitro assay based on human dendritic cells. Toxicol. Sci., 116, 112-129.

Magnusson, B. and Kligman, A.M. (1969): The identification of contact allergens by animal assay. The guinea pig maximization test. J. Invest. Dermatol., 52, 268-276.

Natsch, A. and Emter, R. (2008): Skin sensitizers induce antioxidant response element dependent genes: Application to the in vitro testing of the sensitization potential of chemicals. Toxicol. Sci., 102, 110-119.

Natsch, A., Emter, R. and Ellis, G. (2009): Filling the concept with data: integrating data from different in vitro and in silico assays on skin sensitizers to explore the battery approach for animalfree skin sensitization testing. Toxicol. Sci., 107, 106-121.

Natsch, A., Ryan, C.A., Foertsch, L., Emter, R., Jaworska, J., Gerberick, F. and Kern, P. (2013): A dataset on 145 chemicals tested in alternative assays for skin sensitization undergoing prevalidation. J. Appl. Toxicol., 33, 1337-1352.

Nukada, Y., Miyazawa, M., Saitou, K., Sakaguchi, H. and Nishiyama, N. (2013): Data integration of non-animal tests for the development of a test battery to predict the skin sensitizing potential and potency of chemicals. Toxicol. In Vitro, 27, 609618.

OECD (2012): The adverse outcome pathway for skin sensitization initiated by covalent binding to proteins. Part 1: Scientific evidence. OECD environment, health and safety publications series on testing and assessment. 168, 1-46

Patlewicz, G., Aptula, A.O., Roberts, D.W. and Uriarte, E. (2008): A minireview of available skin sensitisation (Q)SARs/Expert sys- 


\section{In silico risk assessment for skin sensitization}

tems. QSAR Comb. Sci, 27, 60-76.

Patlewicz, G. and Worth, A. (2008): Review of data sources, QSARs and integrated testing strategies for skin sensitization. JRC Scientific and technical reports. EUR $23225 \mathrm{EN}-2008$. http://publications.jrc.ec.europa.eu/repository/handle/111111111/998

Python, F., Goebel, C. and Aeby, P. (2007): Assessment of the U937 cell line for the detection of contact allergens. Toxicol. Appl. Pharmacol., 220, 113-124.

Sakaguchi, H., Ashikaga, T., Miyazawa, M., Yoshida, Y., Ito, Y., Yoneyama, K., Hirota, M., Itagaki, H., Toyoda, H. and Suzuki, H. (2006): Development of an in vitro skin sensitization test using human cell lines; human cell line activation test (h-CLAT). II. An inter-laboratory study of the h-CLAT. Toxicol. In Vitro, 20, 774-784.

Suzuki, M., Hirota, M., Hagino, S., Itagaki, H. and Aiba, S. (2009): Evaluation of changes of cell-surface thiols as a new biomarker for in vitro sensitization test. Toxicol. In Vitro, 23, 687-696.

Takahashi, T., Kimura, Y., Saito, R., Nakajima, Y., Ohmiya, Y.,
Yamasaki, K. and Aiba, S. (2011): An in vitro test to screen skin sensitizers using a stable THP-1-derived IL-8 reporter cell line, THP-G8. Toxicol. Sci., 124, 359-369.

Teubner, W., Mehling, A., Schuster, P.X., Guth, K., Worth, A., Burton, J., van Ravenzwaay, B. and Landsiedel, R. (2013): Computer models versus reality: How well do in silico models currently predict the sensitization potential of a substance. Regul. Toxicol. Pharm., 67, 468-485.

Tsujita-Inoue, K., Hirota, M., Ashikaga, T., Atobe, T., Kouzuki, H. and Aiba, S. (2014): Skin sensitization risk assessment model using artificial neural network analysis of data from multiple in vitro assays. Toxicol. In Vitro, 28, 626-639.

Vacanson, M., Achachi, A., Mutez, V., Cluzel-Tailhardat, M., Varlet, B.L., Rozières, A., Fournier, P. and Nicolas, J.F. (2014): Human $\mathrm{T}$ cell priming assay: depletion of peripheral blood lymphocytes in CD25(+) cells improves the in vitro detection of weak allergen-specific T cells. Experientia Suppl., 104, 89-100. 